J. Clin. Chem. Clin. Biochem.

Vol. 14, 1976, pp. 261-264

\title{
Kinetische Bestimmung des Harnstoffes mit dem LKB-System
}

\author{
Von H.-G. Eisenwiener
}

Laboratorien der Abteilung Diagnostica der F. Hoffmann-La Roche \& Co. AG, Basel

(Eingegangen am 10. Oktober/14. November 1975)

Zusammenfassung: Es wurde die kinetische Bestimmung des Harnstoffes basierend auf der Urease/Glutamatdehydrogenase-Methode für das LKB Reaction Rate Analyzer System 8600 adaptiert. Bei einem Verhältnis von 1:50 zwischen Proben- und Reagenzvolumen wird ein gesicherter Linearitätsbereich bis $33,3 \mathrm{mmol} / \mathrm{l}$ bei einer Präzision von Tag zu Tag von etwa 5\% erhalten. Paralleluntersuchungen wurden sowohl manuell mit der Berthelot/Salicylatmethode als auch auf dem CentrifiChem System mit der Urease/Glutamatdehydrogenase-Methode durchgeführt.

\section{Kinetic determination of urea with the LKB System}

Summary: The kinetic determination of urea based on the urea/glutamate dehydrogenase method was adapted for the LKB Reaction Rate Analyzer System 8600. A ratio of sample to reagent volume of 1:50 ensures linearity up to $33.3 \mathrm{mmol} / \mathrm{l}$ with a day to day precision of $5 \%$. Parallel studies with the urease/glutamate dehydrogenase method were performed with the CentrifiChem System and with the manual Berthelot/Salicylate method.

\section{Einführung}

Bislang wird die Bestimmung des Harnstoffes meistens mit der Urease/Berthelot-Methode bzw. mit der Diacetylmonoxim-Methode ausgeführt. Die Durchführung ist relativ zeitaufwendig und erfordert im allgemeinen 2 Inkubationszeiten bzw. eine Enteiweißung. Nach Spaltung des Harnstoffes mittels Urease bildet der freigesetżte Ammoniak bei der Berthelot-Reaktion über das Chloramin mit Salicylsäure bzw. Phenol ein grünblau gefärbtes Indolderivat; bei der Diacetylmonoxim-Methode bildet der Harnstoff mit Diacetylmonoxim in saurer Lösung einen roten Farbstoff, der photometrisch bestimmt wird. Talke \& Schubert (1) veröffentlichten 1964 die vollènżymatische Bestimmung des Harnstoffes mit Urease und Glutamatdehydrogenase.

Die auch seitens Gutmann \& Bergmeyer (2) ausfuihrlich beschriebene Methode ist eine Endpunktsmethode mit großer Reaktionszeit, nicht ausreichender Linearität bzw. sehr ungünstigem Verhältnis von Probe zu Reagenzvolumen. Diese beiden letzten Punkte lasten auch der bisher veröffentlichten Methode von Haeckel (3), der das Urease/Glutamatdehydrogenase System auf das Gemsaec System adaptierte, an. Die Linearität reicht nur bis etwa $23 \mathrm{mmol} / 1(140 \mathrm{mg} / 100 \mathrm{ml})$ bei dem recht ungünstigen Verhältnis von 1:150 zwischen Probe zu Reagenzvolumen. Durch Erhöhung der NADH-Konzen- tration kann wohl eine geringe, aber keine bedeutend bessere Linearität erreicht werden.

Es wurde festgestellt, daß durch Änderung des bislang meist verwendeten Puffersystems und des $\mathrm{pH}$ sowie durch eine Abstimmung der beiden Enzymaktivitäten von Urease und Glutamatdehydrogenase eine Erweiterung des Linearitätsbereiches bis mindestens $34 \mathrm{mmol} / \mathrm{l}$ $(\simeq 200 \mathrm{mg} / 100 \mathrm{ml})$ möglich ist. Bei dem hier aufgezeigten Reagenzsystem kann mit einem Verhältnis von nur 1:30 zwischen Probe- und Reagenzvolumen gearbeitet werden. Dieses Reagenz bewährte sich sehr gut für die Harnstoff-Bestimmung mit dem CentrifiChem System, wobei $15 \mu \mathrm{l}$ Probenvolumen und nur $350 \mu \mathrm{l}$ Reagenz bei einer Reaktionszeit von nur 1 min eingesetzt wird (4). Aus der Extinktionsdifferenz zwischen der 30. und 90. Sekunde nach dem Mischen wird auf die Harnstoff-Konzentration mit Hilfe eines Standards geschlossen. Es wurde nun versucht, das gleiche Reaktionssystem auf den LKB Reaction Rate Analyzer 8600 zu adaptieren. Bei dem aufgezeigten Verfahren wird mit Glutamatdehydrogenase gestartet, wobei das freie Ammoniak in der Probe miterfaßt wird. Von der Theorie her wäre es günstiger, die Reaktion mit Urease zu starten. Untersuchungen, mit Urease die Reaktion zu beginnen, sind in Vorbereitung, jedoch scheint es günstiger zu sein, nach Beendigung der hydroly- 
tischen Spaltung des Harnstoffes das freigesetzte Ammoniak mit der Glutamatdehydrogenase als initial rate Methode zu bestimmen. Aus den aufgezeigten Versuchsergebnissen kann ersehen werden, daß die Bestimmung des Harnstoffes sehr elegant kinetisch mit einem Rate Analyzer durchgeführt werden kann.

\section{Material und Methoden}

Geräte

LKB Reaction Rate Analyzer 8600

CentrifiChem System

\section{Vergleichsreagenzien}

Für die Paralleluntersuchungen wurden Testpackungen von Diagnostica (F. Hoffmann-La Roche, Basel) eingesetzt: ,Harnstoff (Methode: Urease/Berthelot mit Salicylatreagenż) und ,Harnstoff' für das CentrifiChem System (Methode Urease/Glutamatdehydrogenase). Bei den durchgeführten manuellen und CentrifiChem-Vergleichsuntersuchungen wurden jeweils Proben-Leerwerte mitgeführt.

\section{Apparat-Einstellungen für LKB-System 8600}

Wellenlänge: $340 \mathrm{~nm}$, Meßzeit: $1 \mathrm{~min}$, Meßbereich: 0.050 , Reaktionsverlauf: Decrease, Hintergrund: 0.1, Einspritzvolumen: $50 \mu \mathrm{l}$.

\section{Testkonzentrationen}

$\mathrm{NaH}_{2} \mathrm{PO}_{4} 24 \mathrm{mmol} / \mathrm{l}, \mathrm{Na}_{2} \mathrm{HPO}_{4} 13 \mathrm{mmol} / \mathrm{l}, \mathrm{pH}=6,7,2-\mathrm{O}$ xoglutarsäure $250 \mathrm{mmol} / 1$, Urease (EC 3.5.1.5) >900 U/1, Glutamatdehydrogenase (EC 1.4.1.3) $>1500 \mathrm{U} / 1, \mathrm{NADH}>80$ $\mu \mathrm{mol} / \mathrm{l}$. Die Glutamatdehydrogenase-Lösung der Testpackung wurde mit physiol. $\mathrm{NaCl} 1+19$ verdünnt.

\section{Pipettierung}

$\mathrm{Zu} 1 \mathrm{ml}$ Reagenz werden $20 \mu \mathrm{l}$ Probe pipettiert und nach $15 \mathrm{~min}$ bei $35^{\circ} \mathrm{C}$ mit $50 \mu$ l verdünnter GlutamatdehydrogenaseLösung gestartet.

\section{Berechnung}

Die Berechnung wurde mit einem Standard von $6,67 \mathrm{mmol}$ Harnstoff $/ 1(\triangleq 40 \mathrm{mg} / 100 \mathrm{ml})$ durchgeführt. Eine Berücksichtigung des Reagenzien-Leerwertes erübrigte sich, da er nur innerhalb unserer Auswertegenauigkeit schwankte. Die Änderung des Reagenzien-Leerwertes im Auswerteintervall von 30 s variierte $\mathrm{z}$ wischen 0,0006-0,0008 Extinktionseinheiten. Dieser Unterschied entspricht $0,12-0,16 \mathrm{mmol} / 1$ Harnstoff. Die faktorielle Berechnung ist ebenfalls möglich, wobei der Faktor allerdings empirisch zuvor mit Hilfe einer wäßrigen Verdünnungsreihe zu ermitteln ist, denn es handelt sich um keine Endpunktsreaktion, sondern um eine initial rate Methode. Dabei ist es nicht notwendig, den Harnstoff in eine wäßrige Albumin-Lösung einzuwiegen. Es wird jedoch empfohlen, stets die Berechnung mit Hilfe eines Standards durchzuführen, um eventuell auftretende systematische Änderungen zu berücksichtigen.

\section{Ergebnisse .}

\section{Linearitätsbereich}

Der Linearitätsbereich wurde mit einer wäßrigen Verdünnungsreihe von Harnstoff bis $33,3 \mathrm{mmol} / 1(200 \mathrm{mg} /$ $100 \mathrm{ml}$ ) überprüft. Bei jedem Rack wurde 1 Reagenzien-Leerwert mitgeführt, der jedoch nicht berücksichtigt wurde. Aus Abbildung 1 geht ein linearer Zusammenhang zwischen der gemessenen Extinktionsdiffe= renz pro $30 \mathrm{~s}$ und der Konzentration bis $<33 \mathrm{mmol} / \mathrm{l}$

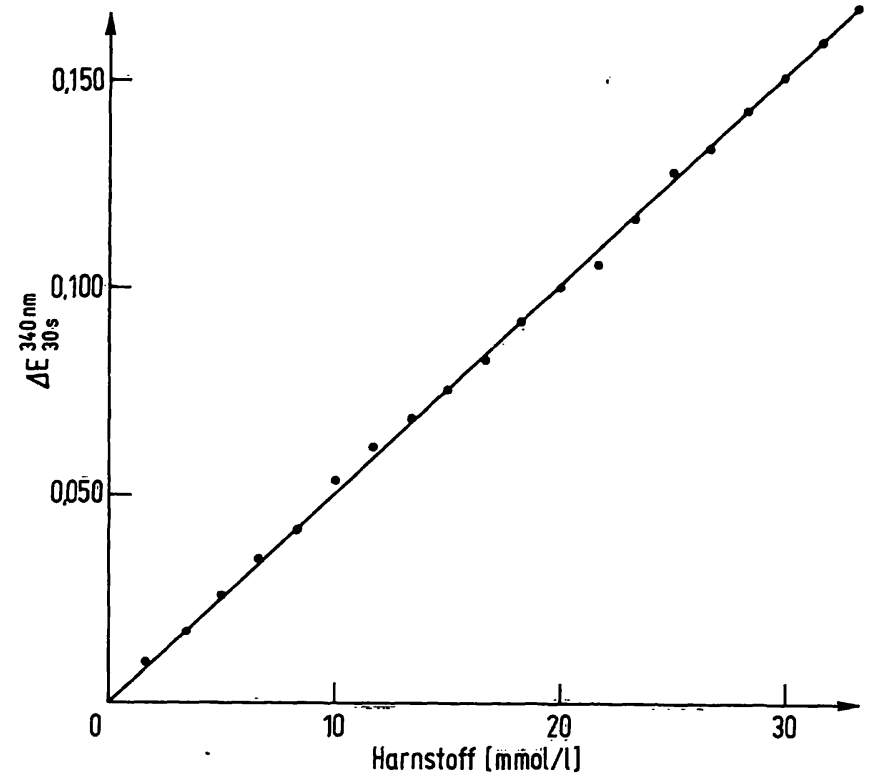

Abb. 1. Linearitätsbereich

( $\simeq 200 \mathrm{mg} / 100 \mathrm{ml}$ ) Harnstoff hervor. Die Bestimmung wurde mit dem Meßbereich (Range) 0.05 bzw. 0.2 durchgeführt. Aus Abbildung $1 \mathrm{kann}$ eine Änderung von 0,083 Extinktionseinheiten pro $30 \mathrm{~s}$ und 16,7 mmol Harnstoff ( $\hat{=} 100 \mathrm{mg} / 100 \mathrm{ml})$ bei einem Verhältnis von 1:50 zwischen Probe: und Reagenzvolumen ersehen werden. Daraus folgt bei einer großzügig angenommenen Auswertungsungenauigkeit von 1 Skalenteil (entspricht 0,0005 Einheiten) ein maximaler Fehler von $0,1 \mathrm{mmol} / 1(0,6 \mathrm{mg} / 100 \mathrm{ml})$.

\section{Richtigkeit}

Die Richtigkeit der kinetischen Harnstoff-Bestimmung wurde mit diversen Kontrollseren überprüft. Aus Tabelle 1 geht eine Gegenüberstellung der gefundenen und der deklarierten Werte hervor. Da die meisten Kontrollseren jeweils in den Einheiten $\mathrm{mg} / 100 \mathrm{ml}$ deklariert sind, wurden die gefundenen Resultate ebenfalls in $\mathrm{mg} / 100 \mathrm{ml}$ angegeben bzw. in $\mathrm{mg} / 100 \mathrm{ml}$ Harnstoff aus $\mathrm{mg} / 100 \mathrm{ml}$ Harnstoff-N umgerechnet. Leider geht im allgemeinen bei den Deklarationen nicht hervor, ob ein Proben-Leerwert bei der Bestimmung mitgeführt wurde.

\section{Präzision}

Die Präzision wurde sowohl mit wäßrigen Lösungen ạ Harnstoff als auch mit Kontrollseren in der Serie $(\mathrm{N}=10)$ und von Tag zu Tag $(\mathrm{N}=20)$ überprüft. Bei den Untersuchungen von Tag zu Tag wurden die lyophilisierten Kontrollseren täglich frisch aufgelöst. Aus Tabelle 2 gehen die erhaltenen Resultate bezüglich Präzision in dêr Serie und von Tag zu Tag hervor. 
Tab. 1. Richtigkeit, Uberprüfung mit Kontrollsera

\begin{tabular}{|c|c|c|c|}
\hline Kontrollserum & Deklarationsmethode & $\begin{array}{l}\text { deklarierte } \\
\text { Werte } \\
{[\mathrm{mg} / 100 \mathrm{ml}]}\end{array}$ & $\begin{array}{l}\text { gefundener } \\
\text { Wert } \\
{[\mathrm{mg} / 100 \mathrm{ml}]}\end{array}$ \\
\hline $\begin{array}{l}\text { Hyland I } \\
0369 \mathrm{GOO} 4 \mathrm{Al}\end{array}$ & $\begin{array}{l}\text { SMA } 12 / 30 \\
\text { SMA } 12 / 60,6 / 60 \\
\text { Hyland: Phenate-hypochlorite } \\
\text { Beckman Rate conductometric } \\
\text { Diacetylmonoxim } \\
\text { BUN, strate } \\
\text { Urograph }\end{array}$ & $\begin{array}{c}32,1 \\
29,9-32,1-34,2 \\
29,9-34,2-38,5 \\
29,9-38,5-47,1\end{array}$ & 32,2 \\
\hline $\begin{array}{l}\text { Hyland II } \\
0368 \text { T } 006 \text { A1 }\end{array}$ & $\begin{array}{l}\text { SMA } 12 / 30 \\
\text { SMA } 12 / 60,6 / 60 \\
\text { Hyland: Phenate-hypochlorite } \\
\text { Beckman Rate conductometric } \\
\text { Diacetylmonoxim } \\
\text { BUN, strate } \\
\text { Urograph } \\
\text { Hycel Mark X }\end{array}$ & $\begin{array}{l}105 \\
101-105-109 \\
90-101-111 \\
88-113-139 \\
116\end{array}$ & 100,6 \\
\hline $\begin{array}{l}\text { Moni-Trol II } \\
\text { Lot } 39 \mathrm{~A}, \mathrm{~B}\end{array}$ & $\begin{array}{l}\text { Roche: Berthelot-Reaktion mod. mit Salicylatreagenz } \\
\text { SMA } 12 / 60 \\
\text { Auto-Analyzer: N-1c } 1 / 11 \\
\text { Merck: DAM Diacetylmonoxim-Reaktion } \\
\text { nach Enteiweißung } \\
\text { Merck: Berthelot; Urease-Spaltung, Ammonium- } \\
\text { bestimmung } \\
\text { Boehringer }\end{array}$ & $\begin{array}{lll}75 & -83 & -91 \\
82,7 & -83,5 & -84,3 \\
80,8 & -86 & -91,3 \\
77 & -86 & -95 \\
77 & -86 & -95 \\
78 & -87 & -96\end{array}$ & 85,8 \\
\hline $\begin{array}{l}\text { Moni-Trol I } \\
\text { Lot } 131 \mathrm{~A}, \mathrm{~B}\end{array}$ & $\begin{array}{l}\text { Roche: Berthelot-Reaktion mod. mit Salicylatreagenz } \\
\text { SMA } 12 / 60 \\
\text { Auto-Analyzer: N-1c } 1 / 11 \\
\text { Merci: DAM Diacetylmonoxim-Reaktion } \\
\text { nach Enteiweißung } \\
\text { Merck: Berthelot; Urease-Spaltung, Ammonium- } \\
\text { bestimmung } \\
\text { Boehringer }\end{array}$ & $\begin{array}{lll}30 & -33 & -36 \\
33,5 & -34,5 & -35,5 \\
33,6 & -36,2 & -38,7 \\
29 & -34 & -39 \\
28 & -33 & -38 \\
30 & -35 & -40\end{array}$ & 35,2 \\
\hline $\begin{array}{l}\text { Pathotrol } \\
66 \mathrm{~A}-\mathrm{Z}\end{array}$ & $\begin{array}{l}\text { DAM Diacetylmonoxim-Reakt. nach Enteiweißung } \\
\text { Berthelot-Urease-Spaltung; } \\
\text { Berthelot-Reaktion }\end{array}$ & $\begin{array}{l}60,5-68,5-76,5 \\
67,0-75,0-83,0 \\
59,5-67,5-75,5\end{array}$ & 75,4 \\
\hline $\begin{array}{l}\text { Labtrol } \\
42 \mathrm{~A}-\mathrm{Z}\end{array}$ & $\begin{array}{l}\text { Berthelot-Reaktion mod. mit Salicylatreagenz } \\
\text { DAM Diacetylmonoxim-Reaktion nach Enteiweißung } \\
\text { Berthelot Urease-Spaltung: Ammoniumbestimmung nach } \\
\text { Berthelot }\end{array}$ & $\begin{array}{l}42-46-50 \\
28,1-32,1-36,1 \\
41,0-46,0-51,0 \\
40.0-45,0-50,0\end{array}$ & 46,4 \\
\hline $\begin{array}{l}\text { Seronorm } \\
118\end{array}$ & $\begin{array}{l}\text { Auto-Analyzer. Diacetylmonoxim-Reaktion } \\
\text { Photometrisch nach Berthelot } \\
\text { Urease/Nessler's Reagenz }\end{array}$ & $\begin{array}{l}53 \\
48,5 \\
51\end{array}$ & 50,3 \\
\hline
\end{tabular}

Tab. 2. Präzision in der Serie $(\mathrm{N}=10)$ und von Tag zu Tag $(\mathrm{N}=20)$

\begin{tabular}{|c|c|c|}
\hline \multicolumn{3}{|l|}{$\begin{array}{l}\text { Präziisionon in der Serie } \\
\text { mit wäßrigen Lösungen }\end{array}$} \\
\hline $\begin{array}{l}\bar{x}=33,3 \mathrm{mmol} / 1(200 \mathrm{mg} / 100 \mathrm{ml}) \\
\bar{x}=16,7 \mathrm{mmol} / 1(100 \mathrm{mg} / 100 \mathrm{ml}) \\
\bar{x} \equiv 1,67 \mathrm{mmol} / 1(10 \mathrm{mg} / 100 \mathrm{ml}) \\
\text { mit Kontrollseren }\end{array}$ & $\begin{array}{l}\mathrm{s}= \pm 0,50 \mathrm{mmol} / 1 \\
\mathrm{~s}= \pm 0,27 \mathrm{mmol} / 1 \\
s= \pm 0,058 \mathrm{mmol} / 1\end{array}$ & $\begin{array}{l}\mathrm{VK}=1,5 \% \\
\mathrm{VK}=1,6 \% \\
\mathrm{VK}=3,4 \%\end{array}$ \\
\hline $\begin{array}{lr}\text { Monitrol I } & 5,46 \mathrm{mmol} / \mathrm{l} \\
\text { Hyland II } & 16,96 \mathrm{mmol} / 1\end{array}$ & $\begin{array}{ll}\mathrm{s}= \pm 0,16 & \mathrm{mmol} / \mathrm{l} \\
\mathrm{s}= \pm 0,40 \mathrm{mmol} / \mathrm{l}\end{array}$ & $\begin{array}{l}\mathrm{VK}=2,93 \% \\
\mathrm{VK}=2,4 \%\end{array}$ \\
\hline Präzisión vọn Tag zu Tag & & $\cdot$ \\
\hline $\begin{array}{l}\text { Monitrol I } 1.31 \mathrm{AB} \\
\bar{x}=5,50 \mathrm{mmol} / 1 \\
\text { Hyland II } 0368 \mathrm{G} \quad 004 \mathrm{A1} \\
\overline{\mathrm{x}}=16,82 \mathrm{mmol} / 1\end{array}$ & $\mathrm{~s}= \pm 0,718 \mathrm{mmol} / \mathrm{l}$ & $\begin{array}{l}\mathrm{VK}=6,4 \% \\
\mathrm{VK}=4,3 \% \\
\end{array}$ \\
\hline
\end{tabular}




\section{Vergleichsuntersuchungen}

Die kinetische Harnstoff-Bestimmung wurde sowohl manuell nach der Berthelot/Salicylat-Methode als auch mit dem LKB und dem CentrifiChem System mit 40 bzw. 60 Seren parallel untersucht und überprüft. Bei der manuellen Methode wurde prinzipiell ein ProbenLeerwert mitgeführt, der sowohl die Eigenabsorption

Tab. 3. Vergleichsuntersuchungen. In 40 Seren wurde parallel, sowohl manuell als auch mit dem LKB und CentrifiChem System, der Harnstoff bestimmt.

manuelle Methode: Urease/Berthelot-Reaktion mit Salicylat LKB + CentrifiChem: kinetische Methode mit Urease/ Glutamatdehydrogenase

$$
\begin{aligned}
& y_{\text {CentrifiChem }}=0,4092+0,9115 x_{\text {manuel }} \\
& \bar{x}=5,79 \mathrm{mmol} / 1 \\
& \overline{\mathrm{y}}=5,69 \mathrm{mmol} / \mathrm{l} \\
& \mathrm{r}=0,982 \\
& \mathrm{n}=40 \\
& y_{L K B}=0,4388+0,9788 x_{\text {CentrifiChem }} \\
& \overline{\mathbf{x}}=5,68 \mathrm{mmol} / \mathrm{l} \\
& \bar{y}=6,01 \mathrm{mmol} / 1 \\
& r=0,980 \\
& \mathrm{n}=40 \\
& \mathrm{y}_{\mathrm{LKB}}=0,7386+0,911 \mathrm{x}_{\text {manuell }} \\
& \overline{\mathrm{x}}=5,79 \mathrm{mmol} / 1 \\
& \bar{y}=6,01 \mathrm{mmol} / \mathrm{l} \\
& r=0,981 \\
& n=40 \\
& y_{L K B}=0,393+0,985 x_{\text {manuell }} \\
& \bar{x}=10,04 \mathrm{mmol} / \mathrm{l} \\
& \overline{\mathrm{y}}=10,29 \mathrm{mmol} / 1 \\
& \mathbf{r}=0,997 \\
& \mathrm{n}=60
\end{aligned}
$$

der Probe als auch die hinzukommende Trübung der Urease-Suspension berücksichtigt. Durch den automatischen Abgleich der Extinktion auf 0 nach Zugabe der Glutamatdehydrogenase und durch Einsatz einer UreaseLösung wird bei dem kinetischen Verfahren eine korrekte Beruicksichtigung des Proben-Leerwertes erreicht. Aus der Tabelle 3 gẹen die erhaltenen Regressionsgeraden, Korrelationskoeffizienten und Mittelwerte hervor. In Abbildung 2 sind die gefundenen Werte auf dem LKB System gegen die manuell ermittelten Werte aufgetragen.

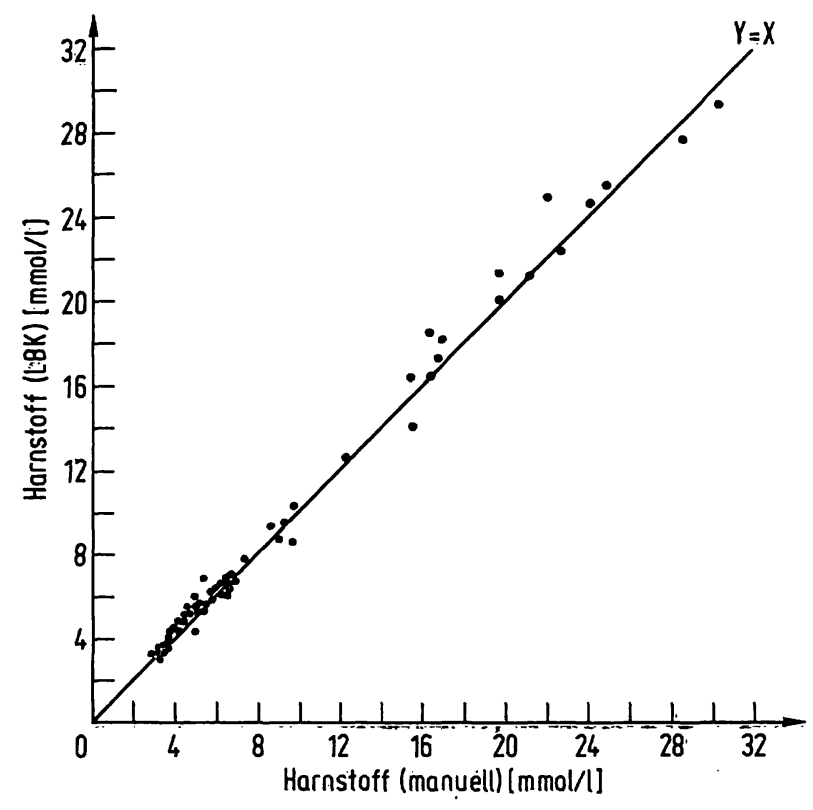

Abb. 2. Vergleichsuntersuchungen

3. Haeckel; R. \& Mathias, D. (1974), diese Z. 12, 515-520.

4. Eisenwiener, H. G. (1975), diese Z. 13, 244-200.

Dr. H.-G. Eisenwiener F. Hoffmann-La Roche \& Co. AG Abt. Diagnostica CH=4133 Schweizerhalle/Basel 